\title{
Insulin therapy for adult patients with type 2 diabetes mellitus: a position statement of the Korean Diabetes Association, 2017
}

\author{
Byung-Wan Lee ${ }^{1,}$, Jin Hwa Kim ${ }^{2,}$, , Seung-Hyun $\mathrm{Ko}^{3}$, Kyu Yeon Hur ${ }^{4}$, Nan-Hee Kim ${ }^{5}$, Sang Youl Rhee ${ }^{6}$, \\ Hyun Jin Kim ${ }^{7}$, Min Kyong Moon ${ }^{8}$, Seok-O Park ${ }^{9}$, Kyung Mook Choi ${ }^{5}$, and on behalf of the Committee of \\ Clinical Practice Guideline of Korean Diabetes Association
}

\begin{abstract}
${ }^{1}$ Division of Endocrinology and Metabolism, Department of Internal Medicine, Yonsei University College of Medicine, Seoul; ${ }^{2}$ Division of Endocrinology and Metabolism, Department of Internal Medicine, Chosun University College of Medicine, Gwangju; ${ }^{3}$ Division of Endocrinology and Metabolism, Department of Internal Medicine, College of Medicine, The Catholic University of Korea, Seoul; ${ }^{4}$ Division of Endocrinology and Metabolism, Department of Internal Medicine, Samsung Medical Center, Sungkyunkwan University School of Medicine, Seoul; ${ }^{5}$ Division of Endocrinology and Metabolism, Department of Internal Medicine, Korea University College of Medicine, Seoul; ${ }^{6}$ Department of Endocrinology and Metabolism, Kyung Hee University School of Medicine, Seoul; ${ }^{7}$ Division of Endocrinology and Metabolism, Department of Internal Medicine, Chungnam National University School of Medicine, Daejeon; ${ }^{8}$ Division of Endocrinology and Metabolism, Department of Internal Medicine, Seoul Metropolitan Government Seoul National University Boramae Medical Center, Seoul; ${ }^{9}$ Department of Internal Medicine, Gwangmyeong Sungae Hospital, Gwangmyeong, Korea
\end{abstract}

Received: September 8, 2017 Accepted: October 14, 2017
The Korean Diabetes Association (KDA) has regularly updated its Clinical Practice Guidelines. In 2017, the KDA published a position statement on the use of antihyperglycemic agents for patients with type 2 diabetes mellitus (T2DM). Growing evidence from new multinational clinical trials using novel and traditional insulin analogues has also been accumulated. Following global trends, many results of clinical trials, especially concerning the clinical efficacy and safety of insulin therapy, have been published about Korean patients with T2DM. After a systematic search of recent evidence, the KDA updated and modified its clinical practice recommendations regarding the initiation, choice, and intensification of insulin and created an insulin treatment algorithm for the first time to guide physicians caring for adult Korean patients with T2DM.

Keywords: Clinical practice guideline; Diabetes mellitus, type 2; Hypoglycemic agents; Insulin; Korea

\section{Correspondence to}

Seung-Hyun Ko, M.D.

Division of Endocrinology and Metabolism, Department of Internal Medicine, College of Medicine, St. Vincent's Hospital, The Catholic University of Korea, 93 Jungbu-daero, Paldal-gu, Suwon 16247, Korea

Tel: $+82-31-249-8155$

Fax: $+82-31-253-8898$

E-mail: kosh@catholic.ac.kr

This manuscript is simultaneously published in the Diabetes Metabolism Journal and the Korean Journal of Internal Medicine by the Korean Diabetes Association and the Korean Association of Internal Medicine.

*These authors contributed equally to this work. 


\section{RECOMMENDATIONS}

\section{Indication of insulin treatment for patients with type 2 diabetes mellitus}

1. Insulin therapy should be initiated if the patient fails to achieve the target glycemic goal despite appropriate treatment with oral hypoglycemic agents [A].

2. Insulin can be used as an initial treatment at the diagnosis of type 2 diabetes in the presence of metabolic decompensation and/or glycosylated hemoglobin $>9.0 \%$ and/or symptomatic hyperglycemia [E].

3. Initiate insulin therapy in the setting of decompensated renal or hepatic insufficiency, myocardial infarction, stroke, acute severe illness, or major surgery $[\mathrm{B}]$.

\section{Choice of type of insulin treatment}

1. A basal insulin regimen or premixed insulin injection (once or twice daily) should be used depending on the patient's circumstances [B].

2. If the glycemic goal is not achieved with a basal insulin or premixed insulin regimen, a multicomponent insulin regimen should be used [A].

3. A combination therapy of oral hypoglycemic agents and insulin can be employed depending on the patient's condition $[\mathrm{A}]$.

\section{WHEN SHOULD INSULIN THERAPY BE INITI- ATED?}

Based on the UK Prospective Diabetes Study (UKPDS) and the Kumamoto study, most of the clinical practice guidelines for glycemic control target of type 2 diabetes mellitus (T2DM) since the early the 2000 s have recommend the achievement of near-normoglycemia to prevent the onset or progression of diabetic microangiopathy $[1,2]$. The progressive nature of $\mathrm{T} 2 \mathrm{DM}$ in the deterioration of pancreatic $\beta$-cell function was such that after 3 years, approximately $50 \%$ of patients could attain glycosylated hemoglobin (HbAic) below $7 \%$ with monotherapy, and by 9 years, this declined to approximately $25 \%$ [1]. Early intensive insulin therapy in patients with newly diagnosed $\mathrm{T} 2 \mathrm{DM}$ has favorable outcomes on the recovery and maintenance of $\beta$-cell function and protracted glycemic remission compared to treatment with oral hypoglycemic agents (OHAs) [3-6]; thus, supporting the rationale for the initiation of insulin therapy in subjects with T2DM whose glycemic targets cannot be achieved with lifestyle modifications and OHAs $[7,8]$. The Korean Diabetes Association recommends insulin therapy in two circumstances: timely initial treatment after the diagnosis of $\mathrm{T}_{2} \mathrm{DM}$ and in cases of $\mathrm{OHA}$ failure.

The initiation of insulin is recommended when severe hyperglycemia (more than $9 \% \mathrm{HbArc}$ ) is detected at diagnosis, especially if hyperglycemic symptoms (polyuria or polydipsia) or any catabolic features (weight loss or ketosis) are present. Insulin should be also considered when adequate glycemic control is not obtained in patients with decompensated hepatic or renal insufficiency and when patients have suffered from myocardial infarction, stoke, or a major operation [9]. For patients with T2DM who fail to achieve the glycemic target with adequate treatment with OHAs, proceed to insulin injection therapy.

\section{HOW TO INITIATE INSULIN THERAPY}

Insulin therapy has the powerful advantage of improving the glycemic control better than other OHAs, but it may be associated with risks of hypoglycemia and weight gain. Therefore, healthcare professionals need to provide comprehensive self-care education including insulin injection skills, self-monitoring of blood glucose, hypoglycemia management, and simple dosage adjustment before patients begin insulin therapy $[8,10]$.

Basal insulin alone or in combination with OHAs is easy to administer and is the preferred choice. Basal insulin including both intermediate-acting and long-acting analogue alone is the most convenient initial insulin regimen. Because long-acting basal analogues (glargine, detemir, and degludec) are evident for reducing the risk of hypoglycemia compared to neutral protamine Hagedorn (NPH) in T2DM, they are preferable to NPH when there is a history of hypoglycemia [11]. Recently, several concentrated basal insulin preparations (U-300 glargine and U-20o degludec) were developed to allow a higher dose of basal insulin injection. Premixed insulin products contain both a basal and prandial component 
(NPH/regular 70/30, 70/30 aspart mix, 75/25, or 50/50 lispro mix), allowing coverage of both basal and prandial needs with a single injection. In the 4 - $T$ study $[12,13]$, patients with T2DM (mean HbA1c 8.5\% and 9-year duration of diabetes at baseline) were randomized to receive premixed insulin twice daily, prandial bolus insulin three times daily, or basal insulin once daily. In this study, median HbAic were comparable after 3 years but weight gain and hypoglycemia were less with basal than with the other insulin regimens. In this study, however, $68 \%$ to $82 \%$ of the patients were taking a second type of insulin at the end of the research period [13]. From meta-analyses, a greater proportion of patients with T2DM achieved the HbA1c goal of $<7 \%$ with biphasic or prandial insulin compared to basal insulin, but these differences were not demonstrated after adjustment for the insulin dose $[14,15]$. The use of premixed or prandial insulin compared to basal insulin was associated with more hypoglycemia and weight gain. Even in previously insulin-treated patients, the frequency of hypoglycemia and weight gain increases as the number of insulin injections increases [15]. The contribution of fasting and postprandial glucose (PPG) on overall hyperglycemia or HbAic is variable among patients. PPG excursions may play a more significant role in patients with early mild to moderate diabetes, whereas fasting hyperglycemia contributes more in patients as diabetes worsens, as represented by increasing concentrations of $\mathrm{HbAlc}$, particularly at $>8.4 \%$ [16]. When physicians initiate insulin therapy, either basal insulin or premixed insulin $[17,18]$, they should understand the pharmacodynamic profile of each formulation and also consider the baseline $\mathrm{HbA1c}$ in conjunction with submaximal doses of sulfonylurea (SU), blood glucose levels in a fasting or postprandial state, and symptoms of hyperglycemia (polyuria or polydipsia).

\section{HOW TO COMBINE ORAL HYPOGLYCEMIC AGENTS WITH INSULIN}

Insulin alone is not an ideal treatment for poorly controlled T2DM, as it is associated with both weight gain and inadequate glycemic control [19]. Various combinations of insulin with OHAs are now available and are widely used [20]. In patients with $\mathrm{T} 2 \mathrm{DM}$ on maximum tolerated OHAs, metformin over placebo decreases weight gain, lowers insulin requirements, and improves glycemic control [19]. From meta-analyses including 2,171 adults with uncontrolled T2DM from 11 randomized controlled trials, adding insulin glargine to metformin monotherapy early in treatment may provide the greatest 24-week reductions in HbAic and less symptomatic hypoglycemia benefits over regimens including SUs [21]. However, the Korean RCT comparing the commonly prescribed OHA combinations (1:1:1 basis with fixed doses of glimepiride, metformin, and glimepiride plus metformin) to use as an add-on therapy with insulin glargine showed a significant improvement in overall glycemic control with insignificant weight gain and the risk of hypoglycemia in the arm of combination therapy of metformin and glimepiride plus glargine insulin [22]. In this post hoc analysis of the Prospective Pioglitazone Clinical Trial in Macrovascular Events study (PROactive), pioglitazone use in combination with insulin resulted in sustained improved glycemic control with a rapid and sustained decrease in insulin doses compared to the placebo group [23]. More insulin-resistant patients (defined as poorly controlled T2DM despite high doses of insulin) in the pioglitazone plus insulin group showed the greatest glycosylated hemoglobin decline [23]. From meta-analyses including 3,092 patients from eight RCTs comparing pioglitazone in combination with any insulin-containing regimen compared to the same insulin regimen alone, pioglitazone confers a small advantage in terms of $\mathrm{HbA}_{1} \mathrm{c}$ in T2DM patients with previous inadequate glucose control but at the cost of increased hypoglycemia and weight gain [24]. Numerous studies of dipeptidyl peptidase 4 (DPP4) inhibitor add-on therapy compared to insulin showed significant improvement in glycemic control relative to the placebo without increasing hypoglycemia or body weight $[15,25,26]$. Sodium glucose cotransporter 2 (SGLT2) inhibitor is a novel insulin-independent OHA that reduces hyperglycemia by reducing proximal renal glucose reabsorption, causing urinary glucose excretion. The adjunctive use of a SGLT2 inhibitor improved glycemic control and reduced weight without increasing the risk of hypoglycemia and with lower insulin requirements, although potential side effects of urinary tract infection and euglycemic diabetes ketoacidosis should be considered [27-29]. In a covariate-adjusted indirect comparison 
using meta-regression analyses including five SGLT2 inhibitors and nine $\mathrm{DPP}_{4}$ inhibitors studies, SGLT 2 inhibitors achieved better glycemic control and greater weight reduction than $\mathrm{DPP}_{4}$ inhibitors without increasing the risk of hypoglycemia in patients with T2DM that is inadequately controlled with insulin [30]. When physicians initiate insulin therapy in patients with $\mathrm{T} 2 \mathrm{DM}$, metformin should be continued while other oral agents may be continued or discontinued on an individual basis, especially insulin regimens to avoid unnecessarily complex or costly OHA regimens.

\section{HOW TO INTENSIFY THE INSULIN THERAPY}

In patients above the HbAic target on basal insulin or premixed insulin once or twice daily, recommendations for further intensification, if needed, are outlined in Fig. 1 [31]. When physicians intensify an insulin regimen, they should consider the advantages and disadvantages such as flexibility, complexity, and frequency of hypoglycemia.

Intensified insulin might consist of dose titration and regimen modification. Once the initiation of an insulin regimen is stable, dose titration for adjusting insulin are made based on the fasting and PPG levels. If a patient is still above the HbArc target with an acceptable fasting blood glucose level on titrated basal insulin, options for treatment intensification are either a single injection of rapid-acting insulin (lispro, aspart, or glulisine) at the largest meal, glucagon-like peptide-1 (GLP-1) receptor agonist (GLP-1RA), or switching to twice daily injections of premixed insulin. These recommendations were based on the non-inferior results of basal insulin + single injection of either rapid-acting insulin or GLP-1RA relative to twice daily premixed insulin [12,13,32-35]. Basal insulin plus GLP-1RA resulted in less hypoglycemia and weight loss compared to other insulin regimens

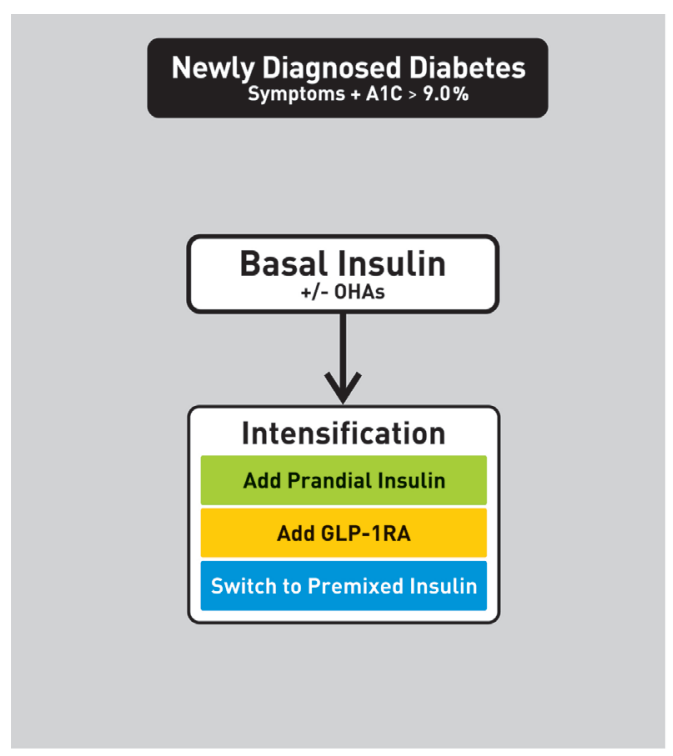

If A1C target is not achieved, consider other regimen at any step. GLP-1RA: GLP-1 receptor agonist

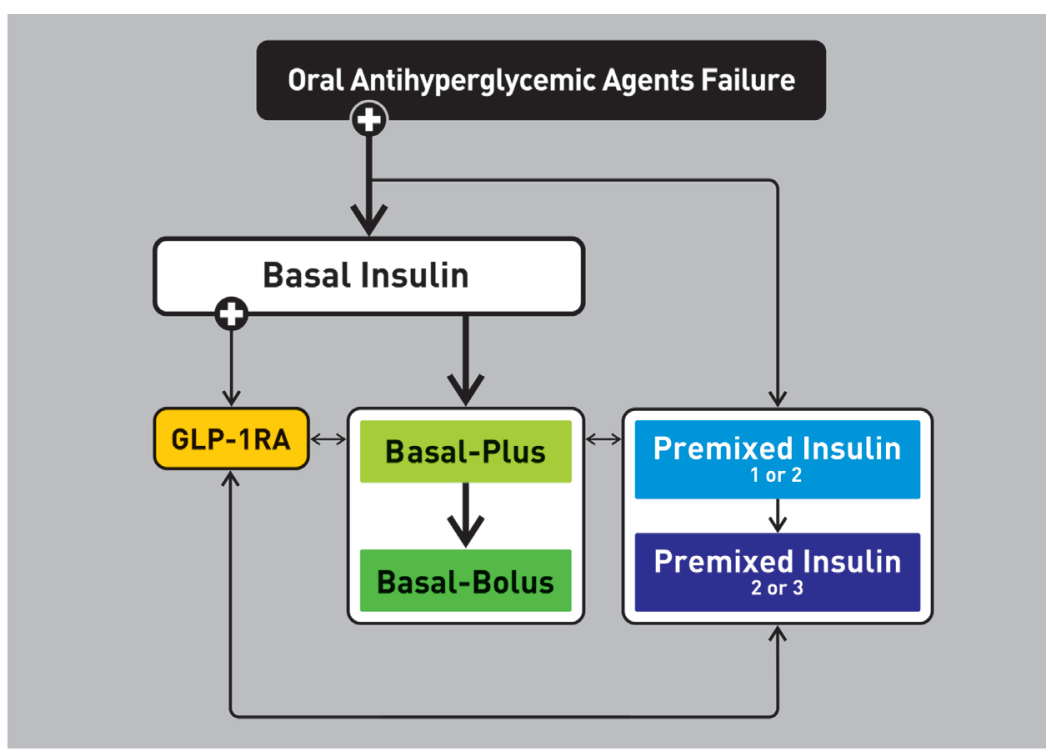

Basal-Plus: add prandial insulin before largest meal Basal-Bolus: add prandial insulin before each meal

Fig. 1. Treatment algorithm for insulin therapy. (A) Initiation of insulin treatment. If the initial glycosylated hemoglobin (ArC) level is $>9.0 \%$ and symptomatic hyperglycemia or metabolic decompensation is present, insulin therapy can be initiated with or without oral antihyperglycemic agents (OHAs) in patients with newly diagnosed type 2 diabetes mellitus (T2DM). If the A1C target range is not achieved after implementing a basal insulin regimen, then proceed to intensification treatment, for example, addition of a glucagon-like peptide 1 receptor agonist (GLP-1RA) or a prandial insulin or switching to a premixed insulin regimen. (B) For adult patients with T2DM who have not achieved their glycemic target following adequate treatment using OHAs. When OHAs fail, proceed to basal insulin either with or without OHAs. The addition of a GLP-1RA or switching to a premixed insulin regimen could be another option depending on the patient's clinical situation. The width of each black line reflects the strength of the expert consensus recommendations. Adapted from Ko et al. [31]. 
[33,34]. If a patient is still above the HbA1c target on basal insulin + a single injection of rapid-acting insulin, naturally advancing to a basal-bolus regimen ( $\geq 2$ times of rapid-acting insulin) should be considered [36]. If a patient is still above the HbAlc target on initial premixed insulin once or twice daily with dose titration, naturally advancing to premixed analog insulin 2 or 3 times daily has been found to be non-inferior to basal-bolus regimens with similar rates of hypoglycemia [17,37]. If a patient is still above the $\mathrm{HbA1c}$ target on an intensified insulin regimen, an option for treatment is switching one regimen with another (such as a basal-bolus regimen to premixed analog insulin three times daily or vice versa) $[38,39]$.

\section{Conflict of interest}

No potential conflict of interest relevant to this article was reported.

\section{Acknowledgments}

Financial support for the development of these guidelines was provided by the Korean Diabetes Association (KDA) operating budget; there was no support or involvement from industry sources.

This position statement on antihyperglycemic agent therapy was written by the KDA Committee of Clinical Practice Guidelines. We gratefully acknowledge the following experts who provided a critical review and discussion of this update: Tae-Nyun Kim, Inje University College of Medicine, Busan; Yong-ho Lee, Severance Hospital, Yonsei University College of Medicine, Seoul; Jin-Hwa Kim, Chosun University Hospital, Gwangju; Eun-Gyoung Hong, Hallym University Dongtan Sacred Heart Hospital, Hwaseong; Jaetaek Kim, Chung-Ang University College of Medicine, Seoul; Won-Young Lee, Kangbuk Samsung Hospital, Sungkyunkwan University School of Medicine, Seoul; Bokrye Song, College of Medicine, Seoul St. Mary's Hospital, The Catholic University of Korea, Seoul; Ji Young Kim, Samsung Medical Center, Sungkyunkwan University School of Medicine, Seoul; Dong Hee Yang, Inje University Ilsan Paik Hospital, Goyang; Taeyoung Yang, Taeyoung 21 Hospital, Gwangju; and Hyeongjin Kim, Kim HJ Medical Clinic, Paju, Korea.

\section{REFERENCES}

1. Turner RC, Cull CA, Frighi V, Holman RR. Glycemic control with diet, sulfonylurea, metformin, or insulin in patients with type 2 diabetes mellitus: progressive requirement for multiple therapies (UKPDS 49). UK Prospective Diabetes Study (UKPDS) Group. JAMA 1999;281:20052012.

2. Ohkubo Y, Kishikawa H, Araki E, et al. Intensive insulin therapy prevents the progression of diabetic microvascular complications in Japanese patients with non-insulin-dependent diabetes mellitus: a randomized prospective 6-year study. Diabetes Res Clin Pract 1995;28:103-117.

3. Weng J, Li Y, Xu W, et al. Effect of intensive insulin therapy on beta-cell function and glycaemic control in patients with newly diagnosed type 2 diabetes: a multicenter randomised parallel-group trial. Lancet 2008;371:1753-1760.

4. Yoo HJ, Park KY, Park KS, et al. Safety and efficacy of modern insulin analogues. Diabetes Metab J 2013;37:181189.

5. Hwang YC, Kang JG, Ahn KJ, et al. The glycemic efficacies of insulin analogue regimens according to baseline glycemic status in Korean patients with type 2 diabetes: sub-analysis from the $\mathrm{A}(\mathrm{1})$ chieve((R)) study. Int J Clin Pract 2014;68:1338-1344.

6. Yang Y, Shin JA, Yang HK, et al. Reduction of sulfonylurea with the initiation of basal insulin in patients with inadequately controlled type 2 diabetes mellitus undergoing long-term sulfonylurea-based treatment. Diabetes Metab J 2016;40:454-462.

7. Kim SS, Kim IJ, Kim YK, et al. Insulin initiation in insulin-naïve Korean type 2 diabetic patients inadequately controlled on oral antidiabetic drugs in real-world practice: the modality of insulin treatment evaluation study. Diabetes Metab J 2015;39:481-488.

8. Choi SH, Oh TJ, Jang HC. Comparison of antidiabetic regimens in patients with type 2 diabetes uncontrolled by combination therapy of sulfonylurea and metformin: results of the MOHAS disease registry in Korea. Diabetes Metab J 2017;41:170-178.

9. Malmberg K, Norhammar A, Wedel H, Ryden L. Glycometabolic state at admission: important risk marker of mortality in conventionally treated patients with diabetes mellitus and acute myocardial infarction: long-term results from the Diabetes and Insulin-Glucose Infusion in Acute Myocardial Infarction (DIGAMI) study. Circulation 
1999;99:2626-2632.

10. Yong YM, Shin KM, Lee KM, et al. Intensive individualized reinforcement education is important for the prevention of hypoglycemia in patients with type 2 diabetes. Diabetes Metab J 2015;39:154-163.

11. Horvath K, Jeitler K, Berghold A, et al. Long-acting insulin analogues versus NPH insulin (human isophane insulin) for type 2 diabetes mellitus. Cochrane Database Syst Rev 2007;(2):CDoo5613.

12. Holman RR, Thorne KI, Farmer AJ, et al. Addition of biphasic, prandial, or basal insulin to oral therapy in type 2 diabetes. N Engl J Med 2007;357:1716-1730.

13. Holman RR, Farmer AJ, Davies MJ, et al. Three-year efficacy of complex insulin regimens in type 2 diabetes. $\mathrm{N}$ Engl J Med 2009;361:1736-1747.

14. Giugliano D, Maiorino MI, Bellastella G, Chiodini P, Ceriello A, Esposito K. Efficacy of insulin analogs in achieving the hemoglobin Alc target of $<7 \%$ in type 2 diabetes: meta-analysis of randomized controlled trials. Diabetes Care 2011;34:510-517.

15. Yki-Jarvinen H, Rosenstock J, Duran-Garcia S, et al. Effects of adding linagliptin to basal insulin regimen for inadequately controlled type 2 diabetes: a $\geq 52$-week randomized, double-blind study. Diabetes Care 2013;36:38753881 .

16. Monnier L, Lapinski H, Colette C. Contributions of fasting and postprandial plasma glucose increments to the overall diurnal hyperglycemia of type 2 diabetic patients: variations with increasing levels of $\mathrm{HbA}(\mathrm{ic})$. Diabetes Care 2003;26:881-885.

17. Aschner P, Sethi B, Gomez-Peralta F, et al. Insulin glargine compared with premixed insulin for management of insulin-naïve type 2 diabetes patients uncontrolled on oral antidiabetic drugs: the open-label, randomized GALAPAGOS study. J Diabetes Complications 2015;29:838-845.

18. Lee YH, Lee BW, Chun SW, Cha BS, Lee HC. Predictive characteristics of patients achieving glycaemic control with insulin after sulfonylurea failure. Int J Clin Pract 2011;65:1076-1084.

19. Douek IF, Allen SE, Ewings P, Gale EA, Bingley PJ; Metformin Trial Group. Continuing metformin when starting insulin in patients with type 2 diabetes: a double-blind randomized placebo-controlled trial. Diabet Med 2005;22:634-640.

20. Suk JH, Lee CW, Son SP, et al. Current status of prescription in type 2 diabetic patients from general hospitals in
Busan. Diabetes Metab J 2014;38:230-239.

21. Fonseca V, Gill J, Zhou R, Leahy J. An analysis of early insulin glargine added to metformin with or without sulfonylurea: impact on glycaemic control and hypoglycaemia. Diabetes Obes Metab 2011;13:814-822.

22. Park CY, Kang JG, Chon S, et al. Comparison between the therapeutic effect of metformin, glimepiride and their combination as an add-on treatment to insulin glargine in uncontrolled patients with type 2 diabetes. PLoS One 2014;9:e87799.

23. Charbonnel B, DeFronzo R, Davidson J, et al. Pioglitazone use in combination with insulin in the prospective pioglitazone clinical trial in macrovascular events study (PROactive19). J Clin Endocrinol Metab 2010;95:2163-2171.

24. Clar C, Royle P, Waugh N. Adding pioglitazone to insulin containing regimens in type 2 diabetes: systematic review and meta-analysis. PLoS One 2009;4:e6112.

25. Hong ES, Khang AR, Yoon JW, et al. Comparison between sitagliptin as add-on therapy to insulin and insulin dose-increase therapy in uncontrolled Korean type 2 diabetes: CSI study. Diabetes Obes Metab 2012;14:795-802.

26. Kothny W, Foley J, Kozlovski P, Shao Q, Gallwitz B, Lukashevich V. Improved glycaemic control with vildagliptin added to insulin, with or without metformin, in patients with type 2 diabetes mellitus. Diabetes Obes Metab 2013;15:252-257.

27. Devineni D, Morrow L, Hompesch M, et al. Canagliflozin improves glycaemic control over 28 days in subjects with type 2 diabetes not optimally controlled on insulin. Diabetes Obes Metab 2012;14:539-545.

28. Rosenstock J, Jelaska A, Frappin G, et al. Improved glucose control with weight loss, lower insulin doses, and no increased hypoglycemia with empagliflozin added to titrated multiple daily injections of insulin in obese inadequately controlled type 2 diabetes. Diabetes Care 2014;37:1815-1823.

29. Wilding JP, Woo V, Soler NG, et al. Long-term efficacy of dapagliflozin in patients with type 2 diabetes mellitus receiving high doses of insulin: a randomized trial. Ann Intern Med 2012;156:405-415.

30. Min SH, Yoon JH, Hahn S, Cho YM. Comparison between SGLT2 inhibitors and $\mathrm{DPP}_{4}$ inhibitors added to insulin therapy in type 2 diabetes: a systematic review with indirect comparison meta-analysis. Diabetes Metab Res Rev 2017;33:e2818.

31. Ko SH, Hur KY, Rhee SY, et al. Antihyperglycemic agent 
therapy for adult patients with type 2 diabetes mellitus 2017: a position statement of the Korean Diabetes Association. Diabetes Metab J 2017;41:337-348.

32. Inzucchi SE, Bergenstal RM, Buse JB, et al. Management of hyperglycemia in type 2 diabetes, 2015: a patient-centered approach: update to a position statement of the American Diabetes Association and the European Association for the Study of Diabetes. Diabetes Care 2015;38:140149 .

33. Diamant M, Nauck MA, Shaginian R, et al. Glucagon-like peptide 1 receptor agonist or bolus insulin with optimized basal insulin in type 2 diabetes. Diabetes Care 2014;37:2763-2773.

34. Eng C, Kramer CK, Zinman B, Retnakaran R. Glucagon-like peptide-1 receptor agonist and basal insulin combination treatment for the management of type 2 diabetes: a systematic review and meta-analysis. Lancet 2014;384:2228-2234.

35. Yoon KH, Hardy E, Han J. Exenatide versus insulin lispro added to basal insulin in a subgroup of Korean patients with type 2 diabetes mellitus. Diabetes Metab J 2017;41:6974 .

36. Raccah D, Bretzel RG, Owens D, Riddle M. When basal insulin therapy in type 2 diabetes mellitus is not enough: what next? Diabetes Metab Res Rev 2007;23:257-264.

37. Giugliano D, Chiodini P, Maiorino MI, Bellastella G, Esposito K. Intensification of insulin therapy with basal-bolus or premixed insulin regimens in type 2 diabetes: a systematic review and meta-analysis of randomized controlled trials. Endocrine 2016;51:417-428.

38. Dieuzeide G, Chuang LM, Almaghamsi A, Zilov A, Chen JW, Lavalle-Gonzalez FJ. Safety and effectiveness of biphasic insulin aspart 30 in people with type 2 diabetes switching from basal-bolus insulin regimens in the A1chieve study. Prim Care Diabetes 2014;8:111-117.

39. Mathieu C, Storms F, Tits J, Veneman TF, Colin IM. Switching from premixed insulin to basal-bolus insulin glargine plus rapid-acting insulin: the ATLANTIC study. Acta Clin Belg 2013;68:28-33. 